\title{
The EV Project Price/Fee Models for Publicly Accessible Charging
}

\author{
Idaho National Laboratory
}

December 2015

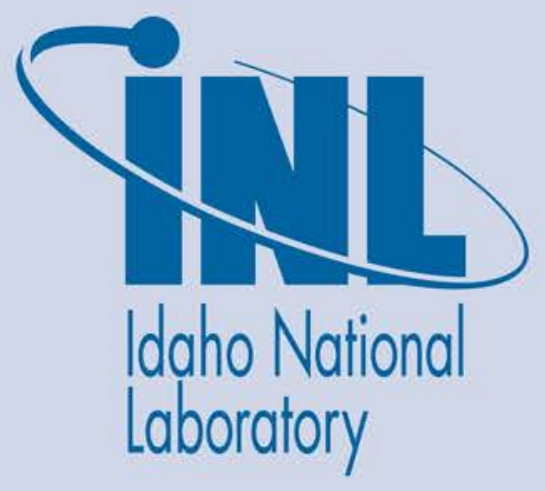

The INL is a U.S. Department of Energy National Laboratory operated by Battelle Energy Alliance 
INL/EXT-15-36314

\title{
The EV Project Price/Fee Models for Publicly Accessible Charging
}

Idaho National Laboratory

December 2015

\begin{abstract}
Idaho National Laboratory
Idaho Falls, Idaho 83415
\end{abstract}

http://www.inl.gov

Prepared for the

U.S. Department of Energy

Assistant Secretary for ____, OR Office of

Under DOE Idaho Operations Office

Contract DE-AC07-05ID14517 


\section{The EV Project Price/Fee Models for Publicly Accessible Charging}

December 2015

\section{Lessons Learned}

- The introduction of fees at AC Level 2 electric vehicle supply equipment (EVSE) did not, over time, reduce charge events at these EVSE, but did reduce the connect time of the average charge event. This was viewed as a positive result. PEVs were getting the same amount of energy, but were no longer parking at chargers for long periods after energy flow had ceased.

- Users generally understood the "need" for fees at publicly accessible chargers, but did debate the appropriate amount of the fee, often based on comparisons to the price of gasoline.

- $\quad$ Guest usage (non-members) at EVSE was higher than initially estimated; generally, guest users navigated the web access or screen instructions without assistance from the call center.

- Hosts desired greater flexibility in use of Blink access cards than expected.

- The host's motivations varied greatly. Some expected revenue sharing and were interested in the return on investment of the EVSE, but many others did not even want the complication of receiving their share of the revenue.

- $\quad$ The project focused on volume (i.e., the more users as a ratio to EVSE the better). Membership and communication helped in this regard.

- Many users relied solely on residential charging.

- Providing visibility through location, internet messaging, and/or signage increased the frequency of charger use.

- $\quad$ There was greater interest in fleets than initially anticipated, although there was also greater complexity in meeting this need.

\section{Introduction}

As plug-in electric vehicles (PEVs) are introduced to the marketplace and gain more consumer acceptance, it is important for a robust and self-sustaining non-residential infrastructure of EVSE to be established to meet the needs of PEV drivers. While federal and state financial incentives for PEVs were in place and remain so today, future incentives are uncertain. In order for PEVs to achieve mainstream adoption, an adequate and sustainable commercial or publicly available charging infrastructure was pursued by The EV Project to encourage increased PEV purchases by alleviating range anxiety and by removing adoption barriers for consumers without a dedicated overnight parking location to provide a home-base charger. This included determining a business model for publicly accessible charge infrastructure.

To establish this business model, The EV Project team created a fee-for-charge model, along with various ancillary offerings related to charging, that would generate revenue. After placing chargers in the field, the project rolled out this fee structure.

The EV Project's model incorporated a combination of fee for charging, advertising, and other supplemental revenue streams to support a privately funded charger infrastructure in public space. The results were not entirely positive, but a fee for charging is both possible and, for several reasons, a good idea. Other revenue streams associated with chargers are sustainable. However, during the term of The EV Project, the combination of these sources of revenue was not sufficient to cover the cost of installing and operating public infrastructure.

\section{Establishing The EV Project Revenue Model}

One of The EV Project objectives was to create a sustainable business model for public charging devices. To do this, the project had to consider who would use public charging, who would host the chargers in public space, what would the costs be, how much would it take to cover those costs, and how to do so. These considerations were integral to all aspects of the project from initial charger design through to the end result of pricing for a charge.

The EV Project deployed over 4,000 alternating current (AC) Level 2 (M2) charging stations and over 100 dual-port direct current fast chargers (DCFC) for commercial use in 17 geographic regions across the United States. Approximately 7,800 Nissan Leaf ${ }^{\circledR}$ and Chevrolet Volt PEVs participated in The EV Project by allowing data to be collected, characterizing operation of their PEVs and their charging usage in exchange for an AC Level 2 EVSE installed by The EV Project at their home location.

The PEV charging stations deployed as part of The EV Project included both residential and non-residential sites. Non-residential sites included EVSE installed in workplace environments, fleet applications, and those that were publicly accessible near retail centers, parking lots, and similar locations. Publicly accessible EVSE were connected via cellular network to the Internet to allow The EV Project to collect charging data; these publicly accessible EVSE were branded Blink ${ }^{\circledR}$. The EV Project rolled out a fee structure for use of publicly accessible Blink EVSE in a 
multi-state effort focused on non-residential charging utilizing a universal price structure with accompanying privileges. The roll-out set pricing for users and hosts with consideration for membership, host return, access rules, fleets, limited access EVSE, rewards, reservations, and advertising as part of a business model intended to develop a sustainable charge infrastructure business.

Success of The EV Project's business model was tied directly to use of non-residential EVSE. The MembershipPrice-Rewards (MPR) Program was intended to encourage use of commercial EVSEs. Initial introductory fees were aggressively low to encourage participation. During the first year, prices were introduced in a relatively simple format. The initial intent was to deploy an easily understood fee and membership program in order to bring the greatest number of users into the program and not confuse a nascent market.

Every portion of the MPR was developed based on its ease of understanding for consumers and hosts. Price, access and other portions of the MPR Program were designed for maximum clarity and the absence of complication. It was understood that the program would evolve and changes would be made, including added complexity.

The EV Project's price program was new and untried; literally at the edge of the "frontier". It was anticipated that experience would provide valuable information, allowing changes based on "real world" use and customer/host feedback. To accomplish meaningful and successful change, The EV Project embraced commentary from users and hosts. Communication programs were established before fees were introduced that encouraged dialogue. Several team members devoted part of each day to stimulating and responding to this dialogue. A member of team leadership reviewed and participated in the dialogue daily using the experience to help design programs and as a part of weekly meeting discussions with the team.

There were a great many questions surrounding the public's need and utilization of non-residential EVSE. The open questions included: (1) what level of fee modifies use, (2) as infrastructure becomes more accessible, will this modify public charging behavior, (3) what would be the average frequency a PEV user would use a commercial EVSE, and (4) would PEVs evolve as short-range vehicles that relied primarily on residential charging. Range anxiety and simple convenience were believed to dictate a need for commercial EVSEs for PEV users. However, how and when non-residential EVSE would be used and how valuable they would be to the PEV user were among a long list of unknowns. By stepping out and introducing a fee for charging, The EV Project began the process of understanding.
A number of related issues impacted how The EV Project established its fee structure, including the following:

- There were not yet enough EV's on the road during the term of The EV Project to build a robust revenue model.

- The cost to fabricate, install, and operate chargers was more significant and costly than was predicted when The EV Project began.

- Hosts were more difficult to attract than anticipated, even when EVSE were provided at no cost to them.

- Local ordinances made permitting in some municipalities difficult and time-consuming.

- Utility demand charges present a difficult cost hurdle.

- Residential chargers were provided by The EV Project at no cost to all participants in exchange for providing charging data from the charger and from their vehicle, assuring that convenient residential charging was available to all participants in The EV Project.

There are substantial costs for providing non-residential EVSE services, including design/fabrication, installation, electricity, service, maintenance, communications, and user authentication costs. In order for non-residential charging to be successful, access fees (or host payment of fees) were required at commercial or publicly accessible EVSE in order to cover at least a portion of the costs, remove freeloader effects of free charging, and provide available charging to many PEV users.

PEV pioneers that participated in The EV Project were provided with an AC Level 2 EVSE that was capable of providing a full charge for a PEV in 4 to 8 hours. The AC Level 2 EVSE was installed at the vehicle's home location. Many of The EV Project participants were able to rely solely on overnight charging utilizing their residential AC Level 2 EVSE to meet their daily driving needs. ${ }^{1}$ This ability to handle daily driving routines without the use of publicly accessible charge infrastructure added to the conundrum of how to make a business out of public charging, when it is not a required source of charging for many PEV drivers.

The EV Project team believed that for public charging to exist on a broad scale with geographic diversity, there must be a significant contribution from private commercial enterprises. Government may play a role in encouraging and expanding public access to charging, but it will not fill the need for broad-based availability of public chargers that is required to provide a viable response to PEV users' range anxiety. To create networks in areas of significant $P E V$ use, a private contribution must be there to augment a government role. 
Why not just government-supplied chargers? At the time of The EV Project, there was a lack of interest on the part of state and local government to make a large investment in infrastructure. While the federal government made a very large investment in charging infrastructure with The EV Project, and the State of California invested in infrastructure for both San Francisco and San Diego, other state governments relied solely on The EV Project for infrastructure deployment.

Why not leave chargers to the vehicle manufacturer? There has recently been a contribution from original equipment manufacturers in support of publicly available chargers. However, during the term of The EV Project, the original equipment manufacturer focus was only on providing a residential charger.

\section{Promoting Use of Publicly Accessible Charge Infrastructure}

A critical factor that concerned The EV Project team and was considered in every aspect of work was getting PEV drivers to use the publicly accessible charge infrastructure. The following factors were of concern to The EV Project team:

- Each participant in The EV Project was provided a residential AC Level 2 EVSE. The convenience of charging overnight at home was a major factor in purchasing a PEV. The convenience of public charging will need to approach that of home charging for significant utilization to occur.

- Purchasers of PEVs were not familiar with using publicly accessible charge infrastructure. This unfamiliarity would be a barrier to use that must be overcome.

- Overnight charging at home is not only convenient, but also inexpensive. The cost of energy at publicly accessible chargers will always be more costly than from residential charging.

- Additional value streams must be ascribed to public charging to make up for the increased cost of energy and installation.

To initially overcome the unfamiliarity with charging away from home, publicly accessible charging was initially offered for free in The EV Project. This also removed any cost issues and incentivized participants in The EV Project to try charging away from home.

Among the many issues involved with a PEV driver utilizing publicly accessible charge infrastructure, the charger location was thought to be the most important. Location showed consistent impact (both positive and negative) on use. There was also believed to be a clear correlation between seeing a charger - "attraction" - and using it. The keys to "attraction" focused on during The EV Project included the following:

1. High volume of vehicle traffic in the parking area

2. High turnover of vehicles in the parking area

3. Visibility of the EVSE

4. Charger located in a desirable parking space

5. No fee or low fee to charge

6. Something for the user to do while charging.

Issues with attraction were obvious, but troublesome. During establishment of the Blink application to support fees for The EV Project, an effort was focused on providing information to help locate publicly accessible charge infrastructure. Many charge locations were found to be difficult to locate, even with the map provided in the Blink mobile device application. A charger located in front of a well-frequented business received more frequent use. A charger located behind a business did not get much use. Interestingly vehicle traffic appeared to have greater influence than free as an attraction. "Being there" was the most important factor, although certainly not a guarantee that the infrastructure would be used. ${ }^{2}$

In establishing a fee model, The EV Project team was concerned that the introduction of fees to a previously free-of-cost charging infrastructure would reduce the use of this publicly accessible charge infrastructure. Once introduced, however, assessing a fee for AC Level 2 charging did not have a significant effect on the frequency of charge events (Figure 1). It did, however, by design, have an impact on the time plugged in (i.e., connect time).

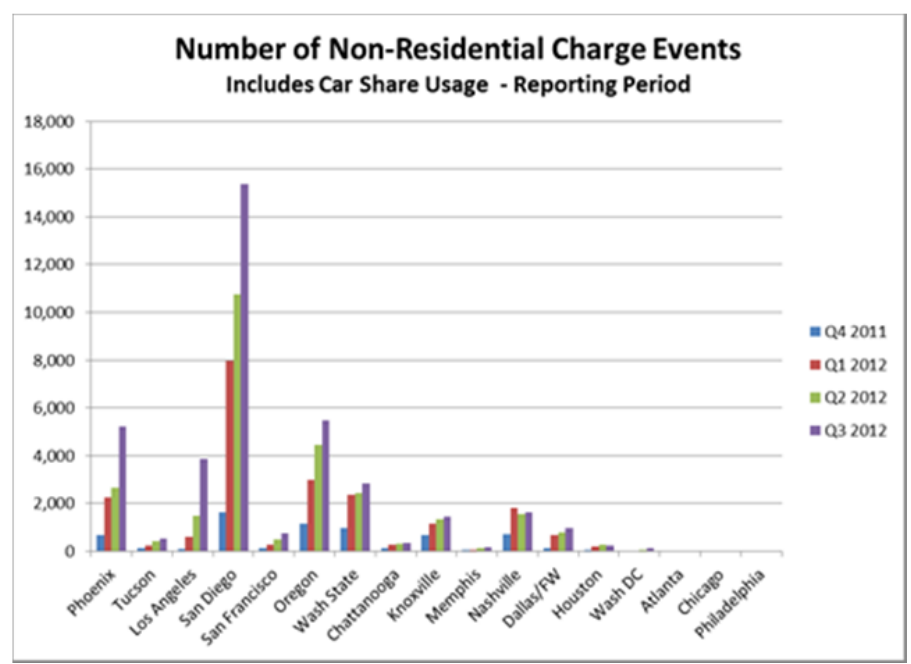

Figure 1. Away-from-home Level 2 charging from the first quarter of 2011 to the third quarter of 2012.

While data from The EV Project's quarterly reports showed that the vast majority of charging was occurring at home, 
rather than through publicly accessible charge infrastructure, ${ }^{3}$ The EV Project team remained focused on trying to build a sustainable charge infrastructure in anticipation that the following factors would, in time, shift more charging to publicly accessible infrastructure.

1. Additional product costs: An AC Level 2 EVSE is an additional purchase cost for a PEV, with a separate manufacturer's suggested retail price between $\$ 300$ and $\$ 2,000$. Future purchasers may opt to not purchase a home EVSE.

2. Expensive installation: Installation of $A C$ Level 2 EVSE at home is an expensive process. The average residential installation costs during the project were between $\$ 800$ and $\$ 2,400 .{ }^{4}$

3. Multi-dwelling units: Multi-dwelling units pose a variety of issues for the installation of AC Level 2 EVSE. The parking spaces are often located in common areas that do not have assigned parking or must be shared equally amongst all users. Costs associated with installation of these charging stations often are not shared by all residences or are difficult to be attributed to one or a few.

4. Street-side parking: PEV drivers that do not have a garage or carport and rely on street-side parking availability may need to rely on public charging $-a$ model more typical of European PEV use (Figure 2).

5. Renters: Renters of properties often will need approval of their landlord/property owner for installation of EVSE. While this is often viewed as a facilities upgrade, many landlords/property owners may be reluctant to provide this installation for short-term or uncertain tenants.

6. Vehicle Leasing: Because many consumers choose to lease their vehicle for 3 to 5 years, there is often uncertainty about future vehicle purchases (may not be a PEV). Consumers who choose to lease a PEV may opt out of residential Level 2 EVSE installation and ownership if ample public charging is available.

As PEVs become more widely adopted, many consumers will face barriers to having a residential or home base AC Level 2 EVSE installed for their vehicle. Consequently, a robust public infrastructure was thought to be necessary to overcome these barriers, alleviate range anxiety (i.e., the "feeling" that one may not be able to fulfill plans due to vehicle range limitations), and make PEV ownership a viable and compelling transportation choice for consumers. The fee model was based on knowledge that, in the short term, profitability was not possible. However, with a model that encourages use of publicly accessible infrastructure, profitability should be possible as the PEV and EVSE populations grew.

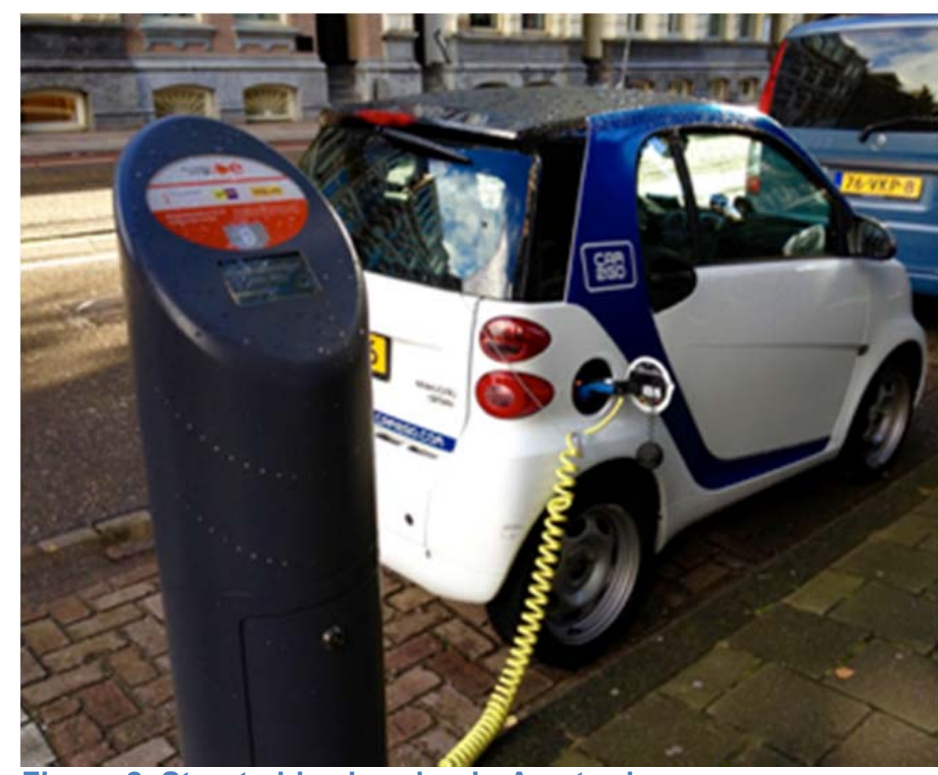

Figure 2. Street-side charging in Amsterdam.

\section{Building the Fee Model}

The EV Project originally surmised that commercial charging in a public space would need to be self-sustaining and generate sufficient revenue to cover the cost of the device, electricity, maintenance, and profit. During the course of the project, it became clear that charging fees alone would not be sufficient to cover costs and generate a profit.

The EV Project team built numerous economic price models using data from the Blink Network (Internet data system collecting charge data from The EV Project chargers), adjusting the number of hours PEVs would need to be plugged into public chargers and projecting what fee was required to produce a viable profit. It was quickly evident that each hour of charging would need to be very expensive to cover the cost of the charger; even when fairly aggressive PEV growth projections were used. Models were constructed comparing the charging fee to the cost of an equivalent mileage operating on gasoline. This fee level was viewed as the upper limit on fees for publicly accessible charging.

Various means of assessing the fees for charging were built into the models. It became clear that a time-based model (pay for connection time) was the simplest and most understandable method. During the timeframe of The EV Project, an electrical consumption model was rolled out in Europe. This was examined for application in The EV Project and, while it was technically feasible (because each Blink EVSE was equipped with a revenue-grade electric meter), re-selling electricity was not allowed in nearly all states at that time. 
The EV Project team made an obligation to find a "business model" for charger providers in a public market and looked at a host of creative ideas to stimulate revenue. The EV Project explored options, including the following:

- $\quad$ Subsidizing non-residential chargers with the sale of residential chargers (not a sustainable concept)

- $\quad$ Advertising and commercial messaging were offered to charger hosts and PEV stakeholders. The EV Project's Blink AC Level 2 EVSE and DCFC were equipped with color screens capable of static and video messaging. This design feature was incorporated to facilitate exploring revenue from advertising on these screens.

- Vinyl wraps on the chargers were developed as an advertising product for a fee.

These and other ideas for promoting hosts were considered and floated to The EV Project hosts to determine if there was a market and what would the market pay.

In conjunction with exploring alternative options, it was necessary to build a fee-based model, determine how that model would work, how users would respond to it, and how much revenue might be reasonably generated. To this end, an elaborate, yet rational, fee model for publicly based chargers was created and implemented.

\section{Elements of the Fee Model}

Summary: The EV Project team created a fully integrated pricing structure to meet its objective of developing a sustainable business for charging infrastructure. Each element of the pricing structure had to be compatible with the capabilities of the Blink charge infrastructure. For example, it was originally anticipated that the fee might be based on each visit or plug in. Once it became clear that users might park all day at a convenient charger, it was decided that the fee needed to be time based to encourage users to make room for the next PEV after "filling up". Changes were required to the existing firmware and software to accomplish this modification in concept. Instructions on how to use the EVSE, including payment for charging, were conveyed to the user through the screen. As changes to the pricing structure occurred, screen content had to be revised and re-tested. The first version of the steps and how they would be communicated did not work well on the screen; they were too verbose.

Modifications to the process and the verbiage were made.

The Fee: Deliberations on the amount of the fee and how to structure the tiers involved extensive debates within The EV Project team. The team extended the discussion of fees to include dialogue with users and hosts to obtain input and feedback on the concepts under consideration. Economic modeling was a major influence on the early design of the fee structure, although a sustainable model for fees was never achieved.

A few basic concepts were relied on to establish the fee structure. First, it needed to be simple and have only a few levels. Second, the price needed to be low, because it was transitioning from free to a fee that should not give rise to "sticker shock," skewing results to The EV Project data. For simplicity, the fee was the same in all seven geographic regions of The EV Project in order to have one universal message and to provide data from a similar base. For several reasons, the project determined that some form of membership would need to be a part of the structure.

Membership: A major challenge to overcome was to get users to charge at publicly accessible chargers.

Membership was thought to be a partial solution to this challenge. Membership was intended to provide a core group of users who would tend to use chargers based on having "joined." A basic membership was established and open to anyone at the cost of charging. A premier membership was offered with a discount on the fee for charging per hour in exchange for a set membership fee, providing the charge infrastructure network an assured monthly revenue. During the duration of The EV Project, participants were provided a premium membership at no cost. One of the major benefits of membership was a means of communicating with users to both learn from their experiences and to offer incentives. Members received regular communications as a Blink member and part of the Blink network. As it turned out, membership solved additional challenges, such as how to authorize use of the charger and obtain fees for charging without use of a credit card at the charger.

The EV Project's engineering team determined, after some effort, that the security requirements for taking credit cards at the EVSE were too onerous to be undertaken. Defined by the Payment Credit Industry Association, these standards included periodic inspection of the card reader to assure that no "piggyback" reader had been attached to divert credit card information. The EV Project determined that this inspection process could not be left to the charger host. Therefore, it would require inspectors from The EV Project to periodically visit each site. The cost of this was prohibitive and an alternative payment solution was sought.

The membership concept was supplemented with a payment process, whereby obtaining membership involved registering a credit card for payment of charging fees. Members received a Blink card that contained a radiofrequency identification (RFID) tag that identified them as a member. The EVSE read the RFID card, verified the member had a current credit card registered, and, based on this, allowed charging to proceed. Fees for charging were then calculated based on the time the PEV was 
connected to the EVSE and charged to the registered credit card.

Guests: The determination that credit cards could not be used at the charger created another challenge. The EV Project was funded by the U.S. Department of Energy (DOE). As such, chargers needed to be broadly available to all who sought to use them, not just Blink members. Therefore, the question of how to deal with those who were not members and did not have a Blink card to activate a charger? A number of ideas were drafted and several were reviewed with engineering to determine which might be possible based on hardware, firmware, and software. The solution required some modifications and relied on a phone call to a call center supporting the Blink network to activate the charger. A separate set of screen instructions was created for guests. Every guest plug-in event was followed up with an invitation to become a member.

Hosts: Input and acceptance by hosts of the fee model was a very important element of the fee design and roll out. Many hosts were anxious that fees be initiated; they readily provided input on how the model ought to be structured. Some hosts viewed fees as a way to cover their costs and generate income. The EV Project team recognized that this cost recovery was not a likely outcome, particularly with the PEV population in 2012. Therefore, efforts to dampen that expectation were a part of the fee roll out. Site hosts with utility-based demand fees for electricity presented a particularly thorny problem that is dealt with separately in another paper. ${ }^{5}$ Some hosts wanted the chargers at their location to be free. Interestingly once the fee was rolled out, some hosts who had originally wanted a fee decided they preferred free. Free introduced its own set of issues: if free, how much should the host pay to support the network and ongoing operation of the EVSE and how would the site deal with users that plugged in and stayed beyond the end of charge, thus obstructing other users from availing themselves of the charger? A monthly flat fee covered the return on the cost of the charger from the host to The EV Project. The problem with "squatting" at the plug was dealt with on a case-by-case basis; often the host could best handle the issue, typically directly with the squatter.

Fleets: In order to recover network operating costs and ongoing EVSE maintenance, the fee concept needed to include fleets and workplace installations. A basic monthly fee was worked out for fleets to cover the costs of the EVSE, installation, and maintenance. The monthly fee varied depending on the amount of support provided to the fleet manager. For example, software was developed to provide fleet managers data on use and various analytical reports. Fleets had the choice of using a membership card for fleet vehicles when not at "home base" or they could pay a monthly fee for the fleet to charge at public chargers.
Advertising: To the extent that advertising could generate revenue, it would alleviate some of the pressure to cover costs with the fee for charging. The EV Project devised several models for advertising to supplement revenue. A vinyl wrap was offered to cover a portion of the charger with a message or logo of an advertiser. This feature had potential value to hosts who had located chargers close to their place of business (Figure 3 ) and to national advertisers interested in the PEV market (Figure 4).

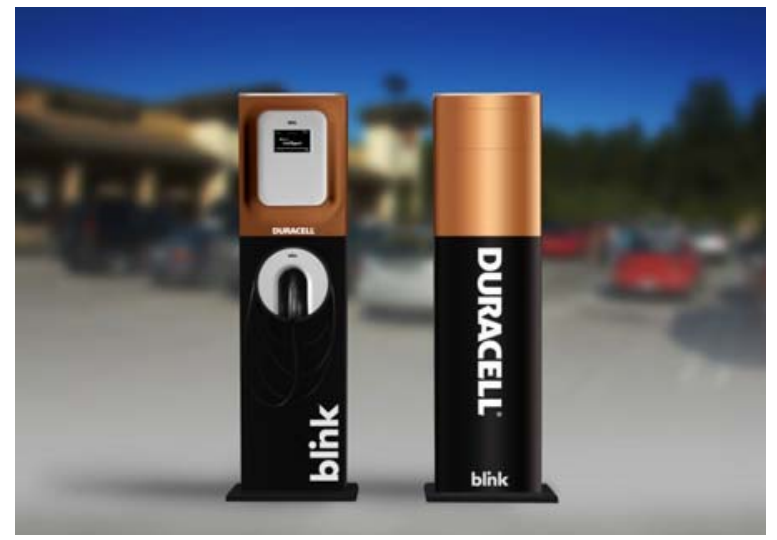

Figure 3. Publicly accessible AC Level 2 EVSE wraps national advertiser.

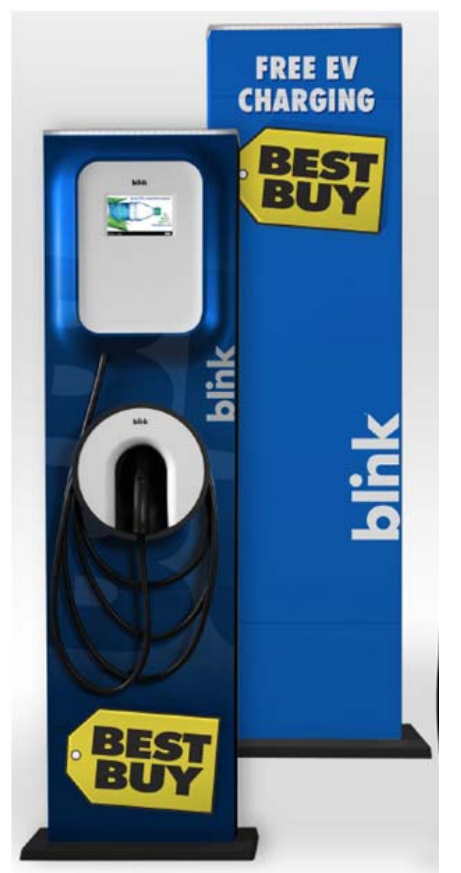

Figure 4. Publicly accessible AC Level 2 EVSE wraps - host advertising.

The charger screen was designed to be able to deliver messages that could accommodate paid-for advertising. The DCFC had a screen dedicated to advertising (Figure 5) and a separate screen provided instructions on charging for 
the user. The AC Level 2 EVSE instruction screen was designed to accommodate both instructions and messaging. The DCFC offered the best option for screen advertising. As can be seen in Figure 5, the DCFC screen was large, making it easily visible to both users and passersby. Additionally, the screens could support advertising, including push advertising providing notice of a host event or sale.

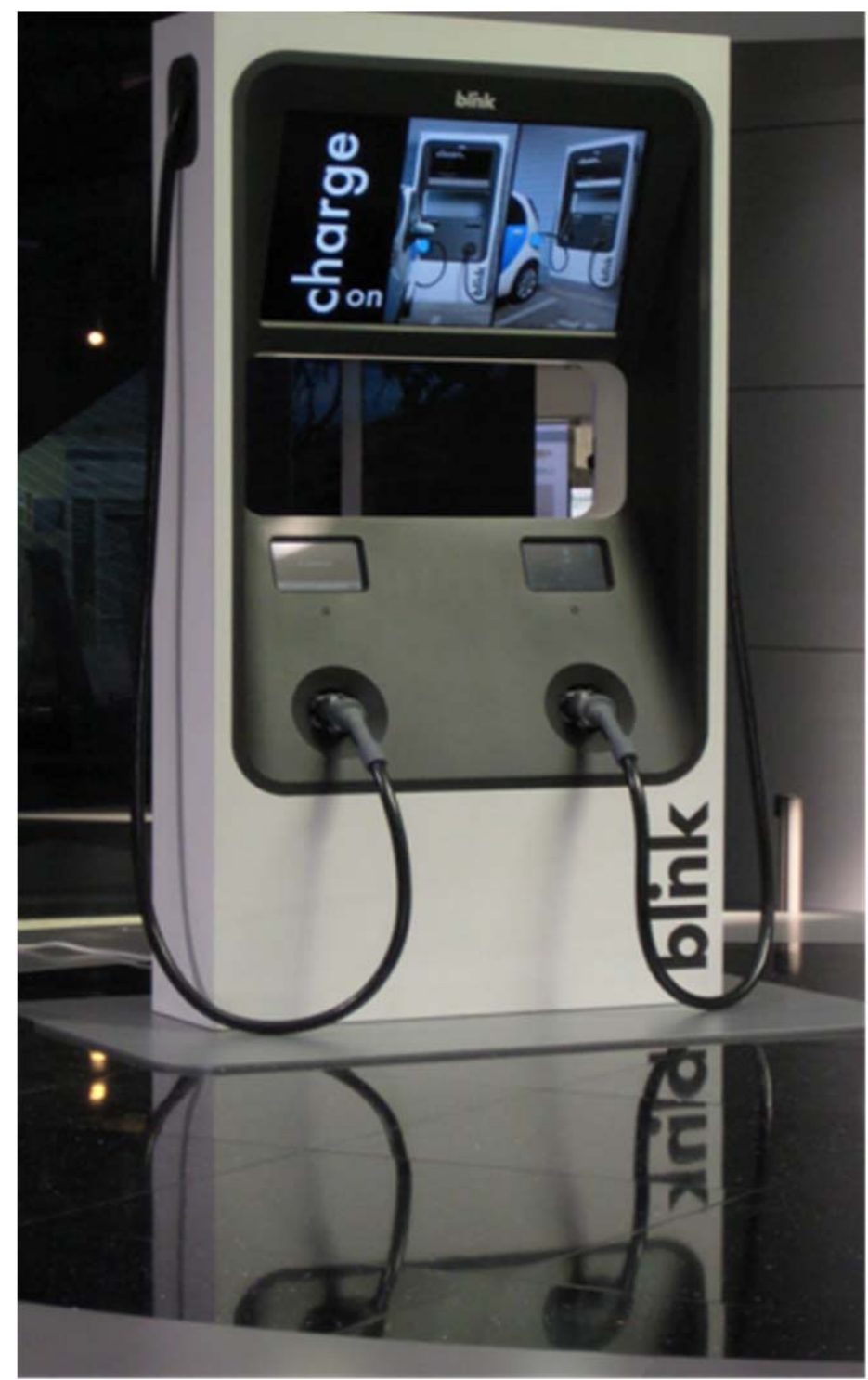

Figure 5. DCFC display screen.

Coupons: Programs were created during the project that were believed to add host value (such as electronic coupons appearing on the EVSE screen that would encourage the PEV driver to visit an adjacent retail business). When this was vetted with The EV Project hosts, the consensus was that this was a good idea, but not worth the effort at the time due to low numbers of PEVs and even lower numbers of PEVs using publicly accessible EVSE.
Other advertising, such as special advertising offers for the host and messages to members highlighting hosts were considered, but not implemented due to low utilization of publicly accessible EVSE.

General advertising on the EVSE screens was also explored. Ad loops were tested and EVSE screens upgraded to better support video. However, advertising placement was not achieved due to the very low number of impressions obtained from publicly accessible charging.

Reservations: A fee program was developed to allow PEV drivers to reserve a particular charger at a specific time. It was felt that this higher assurance that a charger was available would encourage greater PEV use and accompanying greater use of publicly accessible charging. The EV Project established a fully documented program, including supporting software, to allow reservations. A nominal fee for reservations was set, but the reservation program was never rolled out during The EV Project. This was primarily due to unresolved concerns with how to communicate to other PEV drivers that a reservation had been placed on an EVSE for a particular time and the nature of the penalty if another user at an EVSE blocked the reservation.

\section{Implementation of Fees}

Pricing and membership models were designed to accommodate future changes and modification. Some differentiation of pricing between regions was expected. There would also be pricing differences based on site location, either due to the cost of the space or to best accommodate PEV user demand. The critical feature of price was what to sell? Fee for a visit was simple to program into the charger and simple to administer for accounting. However, there were complications with this choice. A fee by visit did not encourage the user to move the vehicle after a given time or when the charge was complete. Thus a vehicle might occupy a charger and not allow another vehicle to use it. During the free period, it was learned that a good deal of host consternation occurred when a PEV occupied space, preventing other customers from charging and visiting the host facility. A number of options were considered to alleviate the extended plug-in scenario. A time-based fee for connect time was thought to be the solution. This is more complex to program into the charger, to administer, and created more questions from users. For example, how much time should be allowed for a grace period before a fee for the next time period is assessed? However, the advantages outweighed the disadvantages; therefore, a time-based fee was used in the first fee roll-out for both AC Level 2 and DCFC (Table 1). 
Table 1. Initial EV Project fee structure.

\begin{tabular}{lcc}
\hline \multicolumn{1}{c}{ Member Class } & AC Level 2 Fee & DCFC Fee \\
\hline Basic & $\$ 2.00 /$ hour & $\$ 5.00 /$ session \\
Premium & $\$ 1.00 /$ hour & $\$ 5.00 /$ session \\
Guest & $\$ 2.00 /$ hour & $\$ 8.00 /$ session \\
\hline
\end{tabular}

\section{Host Revenue}

The EV Project offered to share revenue with most hosts on a 50/50 basis. The decision to do so was made early in the project when there was great emphasis on obtaining host sites for chargers. Even with The EV Project covering most or all of the cost of EVSE installation, it was difficult to secure host sites when few PEVs were visible on the street. Thus, the 50/50 split was agreed to before the full cost to install a charger or frequency of charges per month was known, in order to secure sites for chargers in a timeframe to support planned vehicle deployment. Once it became clear that vehicle sales would roll out in volume later than originally planned and the full cost of design, fabrication, and installation were known, many host agreements were complete and the model was set. It is important to note, that even with basically a free charger and 50/50 access fee revenue split, The EV Project struggled to secure hosts for publicly accessible EVSE. Hosts were reluctant for many reasons, including the following:

- Business disruption during EVSE installation

- Some ill will carryover from the PEV roll out of the late 1990s

- Need for multiple parking spaces to be used to accommodate Americans with Disabilities Act accessibility requirements

- Few PEVs on the road to serve as a customer base.

\section{Impact of Charging Fees}

At the start The EV Project, charging was free. This was intended to provide an incentive for PEV drivers to try publicly accessible charging and become familiar with where chargers were located and how their use could be integrated into the PEV driver's use patterns. It also established a baseline for The EV Project; when fees were implemented, there would be a useful set of comparative data.

Once fees were introduced, the user reaction was very mixed. At first there was the expected confusion and even resentment that charging was not free. For most users this was a brief period that, with the help of a communication to users by The EV Project, evolved to either acceptance and/or understanding. Impacts on charger use from implementation of fees was difficult to separate from the overall growth of charging as more PEV's came into The
EV Project. The EV Project surveyed its participants to obtain their reaction to fees. The results are shown in Figure 6 . The results, in general concluded that (1) some users stopped or reduced public charging due to fees, (2) most did not reduce their frequency of using publicly accessible charge infrastructure (some increased their frequency), and (3) the time plugged-in per visit (i.e., connect time) reduced appreciably. The critical factor for users to frequent publicly accessible charging infrastructure seemed to be that they would not go out of their way to do so. They wanted to charge where they were going to be for an hour or two, but would only go out of their way to charge if there was an actual need to have more range to complete their day and get back home to charge overnight.

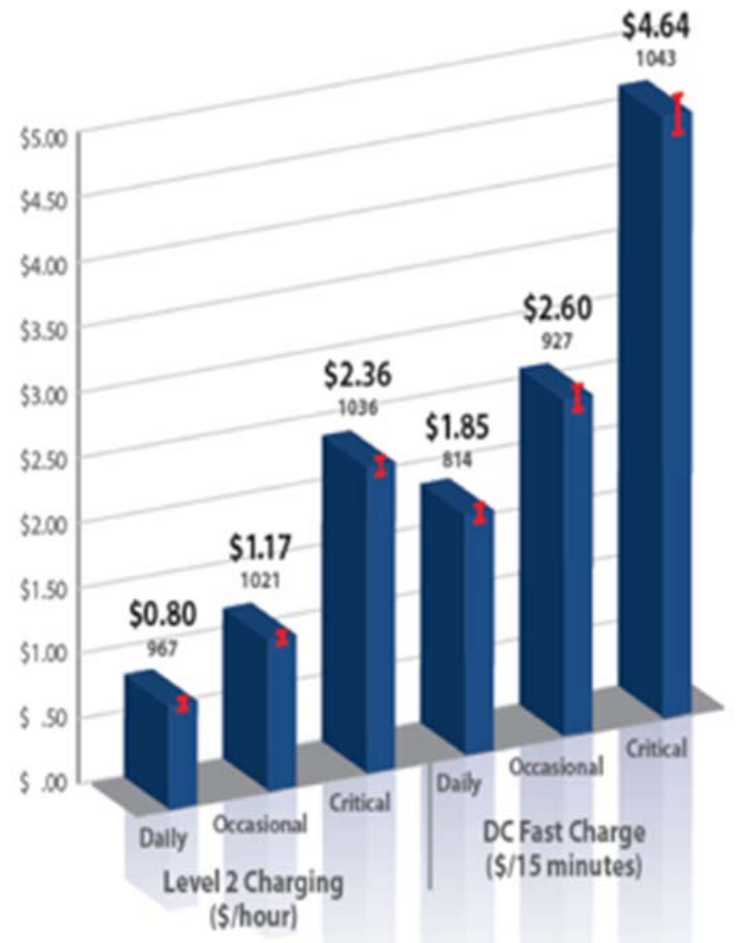

Figure 6. Willingness to pay for AC Level 2 and DCFC charging under three "needs scenarios."

DCFC charging had less price resistance than AC Level 2 EVSE. PEV drivers generally sought out a DCFC when they needed additional range to complete their day. In this situation, a fee was not an obstacle.

\section{Efforts to Encourage Membership}

Membership was important to The EV Project, both as a means of generating more charge events and for enhancing the data collection aspects of The EV Project. The EV Project focused membership on creating a feeling that the member belonged to something special that offered knowledge and value. Building on this concept, The 
EV Project created several programs within membership to encourage participation (see Figure 7). Membership became a structure for increasing charge events, revenue growth, and communication to customers.

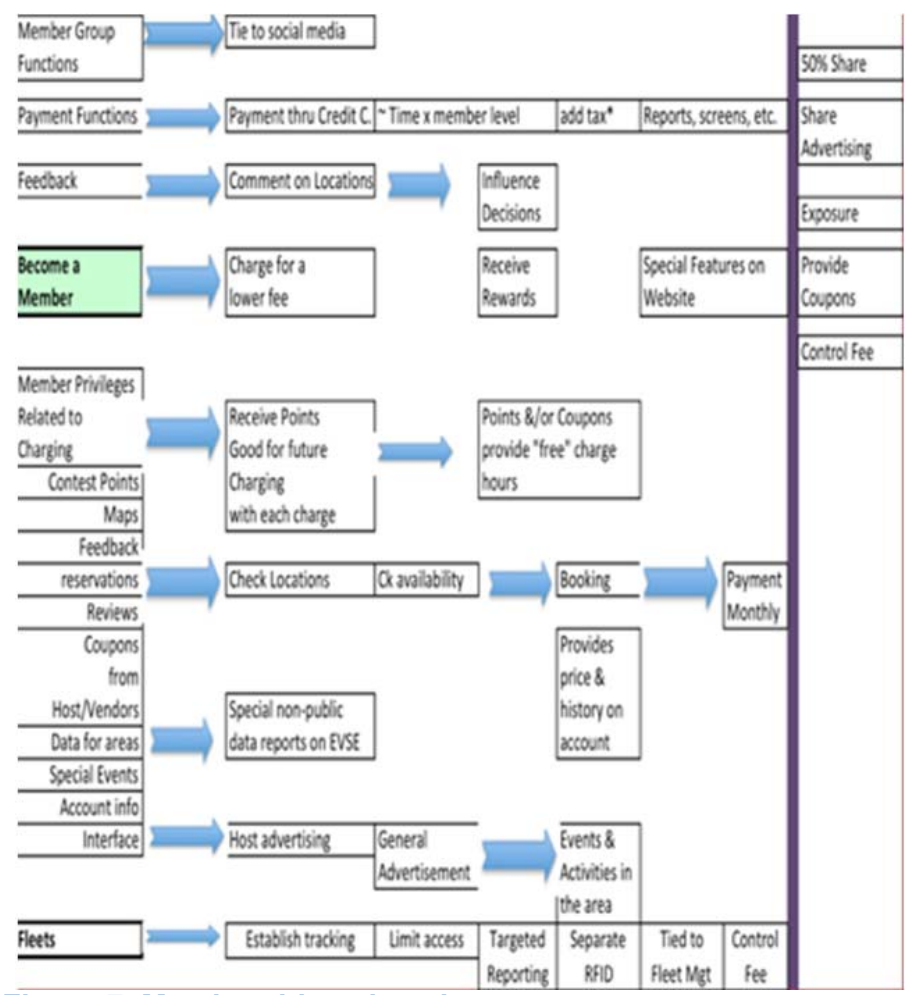

Figure 7. Membership value chart.

\section{Rewards}

The EV Project considered membership rewards to be a contributing piece of the revenue puzzle and means of softening the impact of fees on members who had been charging for free. The evolving rewards program offered charge credits, coupons from merchants, forms of acknowledgement, opportunities for users to recommend charger locations, market data on use, market encouragement, rewards for participation, user contests, and games. Early features included member rewards associated with reduced price and the free $13^{\text {th }}$ hour. Additional rewards for members included contests with rewards and other features to encourage users to value the membership. A rewards program for hosts aimed at both value and belonging was introduced. For both members and hosts, the concept of belonging to the family of EVSE services was believed to be an important part of the PEV experience within The EV Project.

Rewards offered to members during The EV Project included the following:

- Pay less per hour at Blink EVSE

- Receive credit for the free $13^{\text {th }}$ hour
- Data record to Blink Member of charging

- Ability to reserve selected EVSE as a part of the reservations program (not introduced during the project, but acknowledged in correspondence with members)

- Special reports providing data, articles, and white papers

- Participation in online forums

- Participation in meetings, car rallies, and other special gatherings

- Contests and games to encourage involvement and to distinguish the Blink membership

- Optional monthly fee for DCFC use (proposed but never implemented)

- Occasional discounted or free charging to stimulate use

- Host coupons to develop means of demonstrating PEV user interest in charging at the host's EVSE.

\section{BlinkShare}

The EV Project created an online community to encourage potential and existing customers to answer each other's questions and to provide support to help solve problems. The program envisioned the sharing of new ideas on how to improve products and services. It was also a way for sharing information and resources with customers. This was a user-driven site used for communication and to engender customer loyalty.

Aside from technical or support posts, the primary use of BlinkShare was to enable existing and potential customers to engage and interact with each other. The program recognized access to immediate information from the Internet and its social components had power when determining a company's product acceptance. The EV Project believed that user input would provide a means for improving the product user interface and quality. The EV Project also wanted to create a community of PEV users who communicated with each other through The EV Project's site, providing insights to be incorporated in its applications and to provide additional feedback for DOE as a part of The EV Project's grant objectives. Features and benefits of BlinkShare included the following:

1. Allowing open discussion by early-adopting customers who wanted to know about PEVs and infrastructure

2. Contributing to reduced customer support costs by providing an information resource where users could easily access manuals, software bulletins, and other collateral material 
3. Linking to other media outlets of The EV Project such as Facebook, Twitter, Google+, and the Blink Network

4. Enhancing customer service by giving engineering, marketing, and communications teams better insight into customers' concerns, needs, and wants

5. Providing a message window to encourage public charger use, discover locations where users would like to have a public charger, highlight hosts and host programs, and to understand the needs for public charging

6. Receiving feedback on EVSE performance, damaged EVSE, and overall user insights into charger quality.

The EV Project created a number of processes for communication with its users. These included detailed instructions and flow charts. Figure 9 presents an overview of one process for absorbing BlinkShare and other member inputs, trending and analyzing these data, and providing feedback, directly in the case of a large Blink client, and through BlinkShare and other member communications to small Blink clients and members.

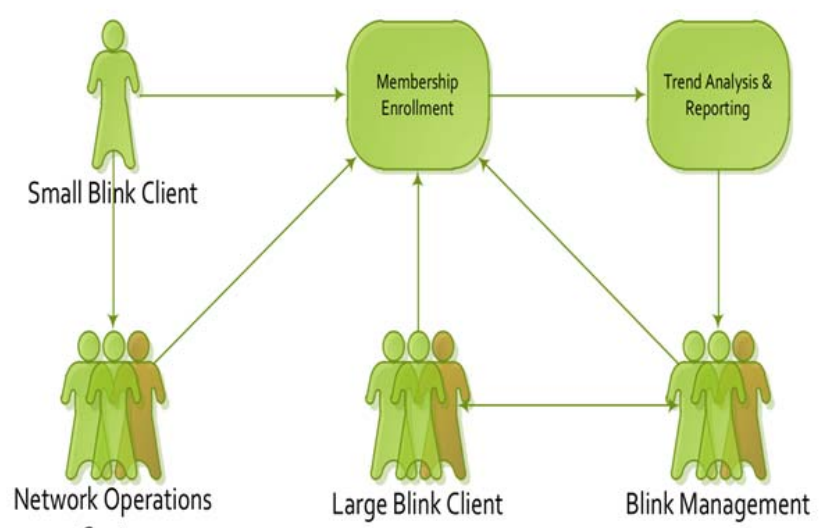

Center

Figure 8. Information sharing flow chart.

\section{Advertising}

Advertising was explored as a means of revenue enhancement. The EV Project experienced mixed success with advertising. The EV Project's charging hardware was specifically designed to enable advertising at the charger. It was believed that this would provide high-value impressions, because the user, their location, and their time of day were all known at the time advertising could be presented. Figure 9 presents the advertising program that The EV Project used to promote electronic advertising on the Blink charging hardware. This program was supplemented by physical advertising in the form of wraps (skinning) (shown in Figures 3 and 4).
Silver Package $(x 2)$ includes

$1 / 5^{\text {th }}$ of the screen time while in screen saver mode

Mention on Blink website and in the mobile app

Gold Package (x2) includes

Includes $1 / 5^{\text {th }}$ of the screen time while in screen saver mode Mention on Blink website and in the mobile app Logo bug in corner of UI listed as sponsor Category exclusivity

Platinum Package $(x 1)$ includes Includes $1 / 5^{\text {th }}$ of the screen time while in screen saver mode Logo bug in corner of Ul during use Mention on Blink website and in the mobile app Category exclusivity Physical Branding rights to chargers where available Electricity sponsorship rights where available

\begin{tabular}{|ccccc|}
\hline Silver & $\begin{array}{c}\text { \# of } \\
\text { Packages }\end{array}$ & $\begin{array}{c}\text { Full Year } \\
\text { Package }\end{array}$ & $\begin{array}{c}\text { Half Year } \\
\text { Package }\end{array}$ & $\begin{array}{c}\text { Quarterly } \\
\text { Package }\end{array}$ \\
\hline Cold & 2 & $\$ 200,000$ & $\$ 130,000$ & $\$ 84,500$ \\
\hline Platinum & 1 & $\$ 1,000,000$ & $\$ 500,000$ & \\
\hline Plat-Electricity & 1 & $\$ 1,000,000$ & $\$ 500,000$ &. \\
\hline Plat-Skinning & 1 & $\$ 1,000,000$ & $\$ 1,000,000$ &. \\
\hline 100\% Sell Through & & $\$ 4,4 m m$ & & \\
\hline
\end{tabular}

Figure 9. The EV Project's advertising program.

Silver and gold programs were proposed in quarterly, half year, and annual packages. Platinum programs were only available in half-year and annual packages. All packages included advertising on $100 \%$ of the available chargers.

The advertising program met with little success, because during the duration of The EV Project, there were insufficient charge events to entice advertisers to use PEV charging as an advertising medium. While The EV Project put a good deal of faith in advertising as a means of creating a revenue stream that would provide sufficient revenue to develop a stand-alone business model, the absence of success does not condemn the concept. It may be that The EV Project team applied a less than ideal method or approach and others in the future will do better. It may also be that The EV Project was conducted in a time period when there were not yet enough PEVs on the road to justify this advertising medium. It is possible that as more PEVs are sold, another organization will realize the returns The EV Project was looking for through direct communication with members and charger users.

\section{The EV Project Revenue Model}

The price points selected for the start of The EV Project's publicly accessible EVSE fee program were universal across all regions. Initially, there were only three price points (Level 2 and DCFC and each had three fees) in order to provide the greatest ease of understanding: 
1. A basic membership for access was provided to anyone who wished to sign up. This basic level allowed users to charge without an annual membership fee. To have this basic service, one had to provide a valid credit card through a web-based account and receive an assigned number for charging.

2. A premium membership, called Blink Plus, required a yearly fee and provided a discounted rate for charging.

3. A guest access mode was designed and implemented to provide an opportunity for all PEV drivers to charge at The EV Project's EVSE. A higher guest access fee covered the additional costs incurred and was intended to encourage sign-up for membership.

\section{Pricing Structure}

Pricing and membership guidelines included flexibility, but within set parameters. There was an expectation that in the future there would be differentiation of pricing between some regions. There was also an expectation that certain EVSE may be at a location that would be better served by premium pricing, either due to the cost of the space or to best accommodate user demand. Charger hosts were expected to adhere to price and membership guidelines established by The EV Project.

1. Hosts and a Premium: The EV Project encouraged hosts to use the prescribed fees indicated above to facilitate use at their EVSE. The EV Project recognized that there would be circumstances where a host would desire a higher fee due to the location of the EVSE or some other factor; they also realized that at some future time, there would be an agreement with the hosts requesting higher fees to allow the "premium" fees under certain circumstances. Membership would be messaged that this extra pricing tier exists once it is in effect at a host location.

2. Regional Differentiation: Regional pricing was not a part of the initial price roll out in 2012. However a regional differentiation was anticipated. The rationale to recognize a regional pricing difference was due to local cost for electricity, siting, land value, permitting fees, and so forth, which vary by region. These differences lead to variations in capital and operating costs by region that ultimately should be reflected in variations in pricing by region. Further, it was anticipated that little inter-region travel would occur with PEVs, minimizing any confusion that might arise from variations in pricing.

3. Power Draw: Prices to accommodate charging power differences between PEVs was considered. The EV Project charged predominately Leaf and Volt PEVs, which at that time both charged nominally at $3.3 \mathrm{~kW}$. It was recognized that once sufficient PEVs became available that charged nominally at $6.6 \mathrm{~kW}$, there would be a need to structure a two-tier pricing structure based on 3.3-kW charging and 6.6-kW charging. This structure would have some flexibility to include EVs that do not precisely match these two levels. The project began preparing for this eventuality but did not implement a fee to cover these circumstances because few 6.6-kW capable PEVs were available during the term of The EV Project.

4. "Free": There were a variety of intended applications that resulted in a " $\$ 0$ " showing on the screen. This designation indicated the user of the EVSE would not have a fee transacted against their account, although clearly charging is not free, because electricity, maintenance, and other costs must be covered. The zero dollars is an indication that either the host or The EV Project had agreed to cover the costs of that charge event. Free units required a member RFID card or a guest code. Several options allowed for free charging under certain circumstances. The free option was an applicable option for hosts (such as fleets and closed campus) that desired to cover the costs and rewards (e.g., $13^{\text {th }}$ hour) of free charging. Free was also applied to circumstances where the EVSE could not authenticate the member's Blink card over the Blink network. The EV Project did not want to turn away users and possibly strand drivers during this early study; therefore, charging was allowed under scenarios where a Blink card was presented and successfully read at the charger, but a communication or other type of error did not allow authorization. The EVSE was programed to allow charging after the third attempt for authorization and not invoice the user.

5. Demand Charges: The EV Project did not implement a means to modify charging in order to reduce or avoid demand charges by a utility. Work was begun on creating a mechanism to modify charging on a DCFC unit to mitigate demand charges. A similar effort was considered, but not undertaken for AC Level 2.

6. Pricing rules: The following set of pricing rules were established for the network and the EVSE:

a. Fee was based on time connected to the PEV (not power flow).

b. A brief grace period was used to avoid consumer conflict.

c. Hosts were expected to follow all guidelines for pricing.

d. Three attempts were made to obtain authorization of the user's Blink card. Following the third attempt, if authorization was not communicated, charge commenced without fee. Later in The EV Project, 
an authorized membership list was downloaded on a routine basis from the Blink Network Operations Center to the publicly accessible EVSE. The EVSE operated using this list for authorization to speed up the authorization process.

e. If the EVSE had not communicated with the Blink Network Operation Center within 7 days, the fees were voided. Usage data from the EVSE were still valid and subsequently collected for reporting and analysis.

f. The Blink Network and the EVSE were coordinated on clock time. The EVSE used a counter system to time the duration of charge events. A grace period was allowed between 1- hour fee periods to avoid any issues with counter accuracy.

g. During the project, a "free" host EVSE paid a negotiated monthly fee for each EVSE, nominally \$25/month/EVSE.

h. Notification of a completed charge was provided to the user.

7. Billing Process: The billing process was implemented by associating each Blink membership card with a valid credit card. This was done during the process of signing up for a Blink card. All transactions were tracked by the Blink card, with billing to the credit card occurring monthly. The credit card charge was communicated to the Blink member by e-mail, when the charge was made. The EV Project chose to give benefit to the customer if a fault or power outage occurred at the EVSE during a charge. Figure 10 presents a diagram of the billing process, with an emphasis on the exceptions resulting from faults during a charge.

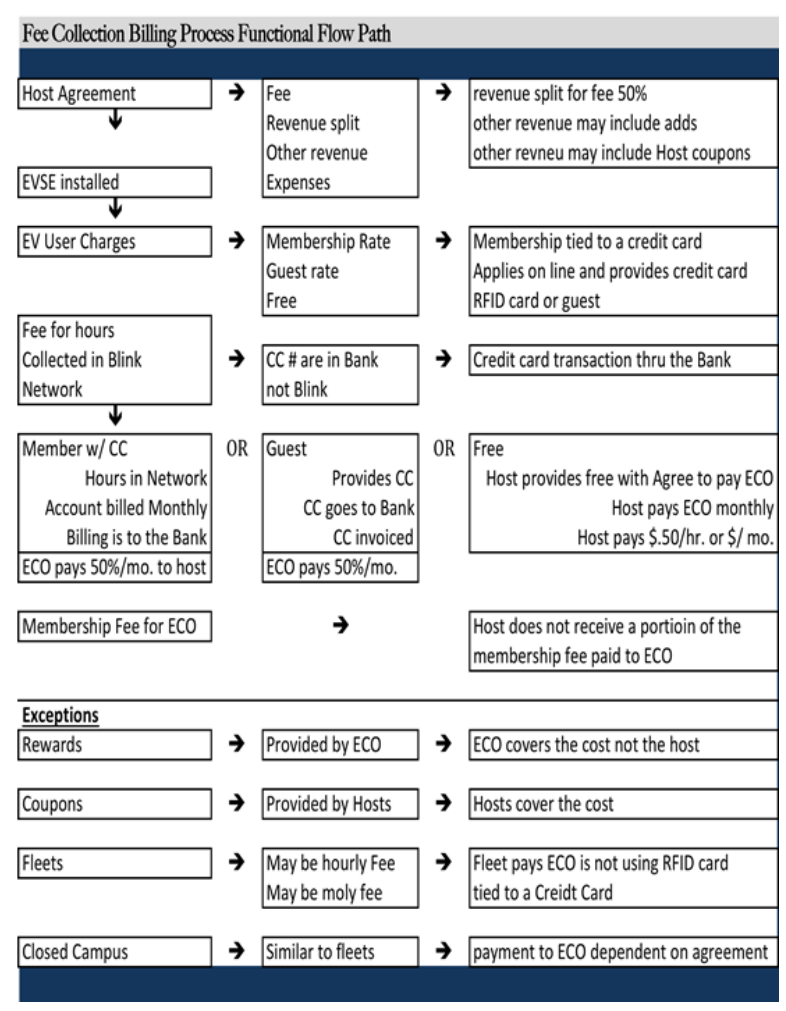

Figure 10. Project fee flow path for billing.

\section{Host Revenue}

1. Commercial hosts revenue and offerings:

a. Level 2 hosts shared in Level 2 revenue at $50 \%$ of gross calculated revenue. Note that DCFC revenue sharing was unique to individual sites.

b. Hosts shared in advertising, reservation fees, and other applications based on agreement.

c. Hosts could participate in coupon programs to encourage EVSE users to visit their business.

d. Hosts were allowed to advertise on their EVSE screens using a content management system developed by The EV Project for host use.

e. Hosts at selected locations were offered the opportunity to wrap the EVSE with their logo or message.

f. Hosts were paid quarterly and could monitor their account online.

g. Hosts could select to provide free charging.

2. Commercial hosts were offered data and reports from their EVSE. 
3. Hosts with multiple EVSE were offered a "VIP" consideration that included early access to promotional programs.

4. Hosts could elect to have their EVSE shut down after normal business hours.

\section{Fee Administration}

The handling of fees involved a number of steps with alternatives all along the way. Documentation was created to define the process with set parameters. The project focused significant effort on training of those who handled accounting or interfaced with others in order to get the best results from users. The project conducted face-to-face, Internet, and conference call training. Following the initial round of training, a weekly call included accounting and service representatives going over unanswered questions and draft answers to user inquiries. The amount of attention given to user communication was believed to be a strength of the project, which was borne out by the short duration of most user challenges and problems.

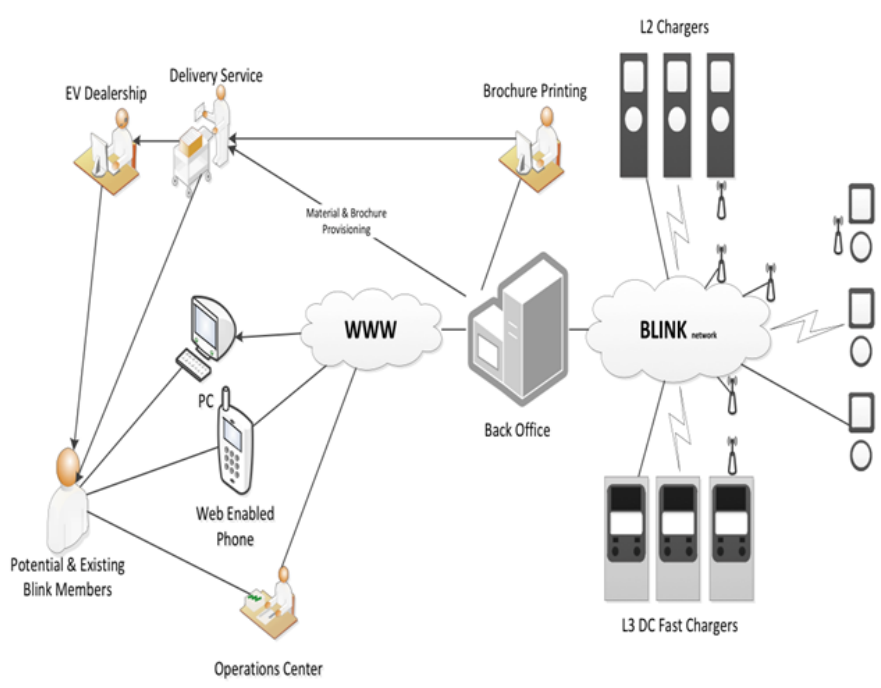

Figure 11. Flow chart from The EV Project defining the process for handling fees.

\section{Conclusions}

The concepts of pricing for users and payment to hosts involved a complex set of issues, including consideration for membership, host return on investment, access rules, fleets, limited access, rewards, reservations, advertising, and data reporting. The success of the price-based model was closely tied to the growth of PEVs and their use of publicly accessible charging. Price/fee programs were developed by The EV Project based on providing ease of understanding and use for consumers and hosts. Price, access, and other aspects were designed for maximum clarity and the absence of complication because they were early in PEV deployment. It was understood that The EV Project was at the edge of the "frontier" and that there was a lot to learn from this first application.

Creating a fee-based system is a complex process that involves many different and sometimes competing elements. The charger itself, even if designed from the start for fees, will have limitations that will dictate some of the decisions on fees. This also held true in The EV Project for both firmware and software. Utilities had a strong influence on the level of fees due to demand charges and rate structures. Of course the user and host played a key role in helping to define the structure for applying and collecting fees.

The introduction of fees worked smoothly during The EV Project. Users were initially loud in voicing their concern that free had ended, but then quickly settled into using the chargers at about the same rate they had without fees.

The results of modeling fees to match costs were disappointing. The EV Project's experience with fees and extensive economic modeling did not provide a sustainable business plan for building out a large, geographically diverse charging infrastructure. It may be that there are approaches that will work by attracting more PEV drivers to use publicly accessible charging infrastructure. It may also be that a much larger population of PEVs will provide the additional charger utilization that is necessary to move toward a profitable model. Additional value streams, such as advertising, provide an opportunity to move to profitability more rapidly.

\section{About The EV Project}

The EV Project was the largest PEV infrastructure demonstration project in the world, equally funded by DOE through the American Recovery and Reinvestment Act and private sector partners. The EV Project deployed over 12,000 AC Level 2 charging stations for residential and non-residential use and over 100 dual-port DCFCs in 17 U.S. regions. Approximately 8,300 Nissan Leafs ${ }^{\mathrm{TM}}$, Chevrolet Volts, and Smart ForTwo Electric Drive vehicles were enrolled in the project.

Project participants gave written consent for EV Project researchers to collect and analyze data from their vehicles and/or charging units. Data collected from the vehicles and charging infrastructure represented almost 125 million miles of driving and 4 million charging events. The data collection phase of The EV Project ran from January 1 , 2011, through December 31, 2013. Idaho National Laboratory is responsible for analyzing the data and publishing summary reports, technical papers, and lessons learned on vehicle and charging unit use. 


\section{Company Profile}

Idaho National Laboratory is one of DOE's 10 multi-program national laboratories. The laboratory performs work in each of DOE's strategic goal areas: energy, national security, science, and the environment. Idaho National Laboratory is the nation's leading center for nuclear energy research and development. Day-to-day management and operation of the laboratory is the responsibility of Battelle Energy Alliance.

For more information, visit avt.inl.gov/evproject.shtml and avt.inl.gov/chargepoint.shtml.

\section{References}

${ }^{1}$ “'Characterize Plug-In Electric Vehicle Driver Away-FromHome Parking Behavior in The EV Project," http://avt.inl.gov/evproject.shtml - LessonsLearned.

2"'Do Plug-In Electric Vehicle Drivers Park near Publicly Accessible EVSE in San Diego but Not Use Them?," http://avt.inl.gov/evproject.shtml - LessonsLearned.

${ }^{3}$ Ibid.

${ }^{4}$ “How do Publicly Accessible Charging Infrastructure Installation Costs Vary by Geographic Location?," http://avt.inl.gov/evproject.shtml - LessonsLearned.

${ }^{5}$ "Characterize the Demand and Energy Characteristics of Non-Residential Alternating Current Level 2 Electric Vehicle Supply Equipment.,"

http://avt.inl.gov/evproject.shtml - LessonsLearned. 